Proyecciones Journal of Mathematics

Vol. 40, No 5, pp. 1341-1356, October 2021.

Universidad Católica del Norte

Antofagasta - Chile

\title{
Unit groups of group algebras of abelian groups of order 32
}

\author{
Suchi Bhatt \\ M. M. M. University of Technology, India \\ and \\ Harish Chandra \\ M. M. M. University of Technology, India \\ Received : August 2020. Accepted : April 2021
}

\begin{abstract}
Let $F$ be a finite field of characteristic $p>0$ with $q=p^{n}$ elements. In this paper, a complete characterization of the unit groups $U(F G)$ of group algebras $F G$ for the abelian groups of order 32, over finite field of characteristic $p>0$ has been obtained.
\end{abstract}

Key words: Group algebras, Unit groups, Jacobson radical.

Mathematics Subject Classification (2010): 16S34, $17 B 30$. 


\section{Introduction}

Let $F G$ be the group algebra of a group $G$ over a field $F$. Suppose $U(F G)$ be the group of all invertible elements of the group algebra $F G$, called unit group of $F G$. In this paper, we study the unit groups of group algebra for abelian groups of order 32. Suppose $V(F G)$ be the normalized unit group, $\omega(G)$ be the augmentation ideal of $G, J(F G)$ is the Jacobson radical of the group algebra and $V=1+J(F G)$. It is known fact that $U(F G) \cong$ $V(F G) \times F^{*}$. An element $g \in G$ is called $p$-regular if $(p, o(g))=1$, where Char $F=p>0$. Notation used in this paper are same as in [2]. Our problem is based on the Witt-Berman theorem [6, Ch.17, Theorem 5.3], which states that the number of non-isomorphic simple $F G$-modules is equal to the number of $F$-conjugacy classes of $p$-regular elements of $G$. Problem of finding unit groups of group algebras generated a considerable interest in recent decade and can be easily seen in [5, 7, 8, 10, 13-15]. Recently in $[1,12]$, Sahai and Ansari have characterized the unit groups of group algebras of groups of orders 16 and 20. Let $G$ be a group of order 32 , we have seven non-isomorphic abelian groups $C_{32}, C_{16} \times C_{2}, C_{8} \times C_{4}$, $C_{8} \times C_{2}^{2}, C_{4}^{2} \times C_{2}, C_{4} \times C_{2}^{3}$ and $C_{2}^{5}$. Here, we have obtained the structure of the unit groups of the group algebras for all these groups over any finite field of characteristics $p>0$. We denote $G L(n, F)$ the general linear group of degree $n$ over $F, M(n, F)$ the algebra of all $n \times n$ matrices over $F, C h a r F$ the characteristic of $F, C_{n}$ is the cyclic group of order $n$ and $F^{*}=F \backslash\{0\}$.

\section{Preliminaries}

Following are the important results which we have used frequently.

Lemma 1. [4, Proposition 1.2] The number of simple components of $F G / J(F G)$ is equal to the number of cyclotomic $F$-classes in $G$.

Lemma 2. [3, Lemma 2.1] Let $F$ be a finite field of characteristic $p$ with $|F|=q=p^{n}$. Then $U\left(F C_{p}^{k}\right)=C_{p}^{n\left(p^{k}-1\right)} \times C_{p^{n}-1}$.

Lemma 3. $[9$, Lemma 2.3] Let $F$ be a finite field of characteristic $p$ with $|F|=q=p^{n}$. Then

$$
U\left(F C_{p^{k}}\right) \cong \begin{cases}C_{p}^{n(p-1)} \times C_{p^{n}-1} & \text { if } k=1 \\ \prod_{s=1}^{k} C_{p^{s}}^{h_{s}} \times C_{p^{n}-1}, & \text { otherwise }\end{cases}
$$

where $h_{k}=n(p-1)$ and $h_{s}=n p^{k-s-1}(p-1)^{2}$ for all $s, 1 \leq s<k$. 
Lemma 4. [11] Let $G$ be a group and $R$ be a commutative ring. Then the set of all finite class sums forms an $R$-basis of $\zeta(R G)$, the center of $R G$.

Lemma 5. [11] Let $F G$ be a semisimple group algebra. If $G^{\prime}$ denotes the commutator subgroup of $G$, then

$$
F G=F G_{e_{G^{\prime}}} \oplus \Delta\left(G, G^{\prime}\right)
$$

where $F G_{e_{G^{\prime}}} \cong F\left(G / G^{\prime}\right)$ is the sum of all commutative simple components of $F G$ and $\Delta\left(G, G^{\prime}\right)$ is the sum of all the others.

\section{Main Results}

Theorem 1. Let $F$ be a finite field of characteristic $p>0$, having $q=p^{n}$ elements and $G \cong C_{32}$.

1. If $p=2$. Then,

$$
U\left(F C_{32}\right) \cong C_{32}^{n} \times C_{16}^{n} \times C_{8}^{2 n} \times C_{4}^{4 n} \times C_{2}^{8 n} \times C_{2^{n}-1} .
$$

2. If $p \neq 2$. Then,

$$
U\left(F C_{32}\right) \cong \begin{cases}C_{p^{n}-1}^{32}, & \text { if } q \equiv 1 \bmod 32 ; \\ C_{p^{n}-1}^{2} \times C_{p^{2 n}-1}^{15}, & \text { if } q \equiv-1 \bmod 32 ; \\ C_{p^{8 n}-1}^{2} \times C_{p^{4 n}-1}^{2} \times C_{p^{2 n}-1}^{3} \times C_{p^{n}-1}^{2}, & \text { if } q \equiv 3,-5,11,-13 \bmod 32 ; \\ C_{p^{8 n}-1}^{2} \times C_{p^{4 n}-1}^{2} \times C_{p^{2 n}-1}^{2} \times C_{p^{n}-1}^{4}, & \text { if } q \equiv-3,5,-11,13 \bmod 32 ; \\ C_{p^{n}-1}^{2} \times C_{p^{4 n}-1}^{4} \times C_{p^{2 n}-1}^{7}, & \text { if } q \equiv 7 \bmod 32 ; \\ C_{p^{n}-1}^{8} \times C_{p^{2 n}-1}^{4} \times C_{p^{4 n}-1}^{4}, & \text { if } q \equiv-7 \bmod 32 ; \\ C_{p^{n}-1}^{2} \times C_{p^{2 n}-1}^{15}, & \text { if } q \equiv 15 \bmod 32 ; \\ C_{p^{n}-1}^{16} \times C_{p^{2 n}-1}^{8}, & \text { if } q \equiv-15 \bmod 32 .\end{cases}
$$

Proof. The presentation of $C_{32}$ is given by

$$
C_{32}=<a \mid a^{32}=1>\text {. }
$$

1. If $p=2$, then $|F|=q=2^{n}$. Since $G \cong C_{32} \cong C_{2^{5}}$, therefore using Lemma 3, we have

$$
U\left(F C_{32}\right) \cong C_{32}^{n} \times C_{16}^{n} \times C_{8}^{2 n} \times C_{4}^{4 n} \times C_{2}^{8 n} \times C_{2^{n}-1} .
$$


2. If $p \neq 2$, then $p$ does not divides $\left|C_{32}\right|$, therefore by Maschke's theorem, $F C_{32}$ is semisimple over $F$. Hence by Wedderburn decomposition theorem and by Lemma 5 , we have

$$
F C_{32} \cong\left(\bigoplus_{i=1}^{r} M\left(n_{i}, D_{i}\right)\right)
$$

where for each i, $n_{i} \geq 1$ and $D i$ 's are finite field extensions of $F$. Since group is abelian, therefore dimension constraint gives $n_{i}=1$, for every $i$. It is clear that $C_{32}$ has 32 conjugacy classes. Now for any $k \in N, x^{q^{k}}=x, \forall x \in \zeta\left(F C_{32}\right)$ if and only $\widehat{C_{i}^{q^{t}}}=\widehat{C_{i}}$, for all $1 \leq i \leq 32$. It exists if and only if $32 \mid q^{k}-1$ or $32 \mid q^{k}+1$. If $D_{i}^{*}=<y_{i}>$ for all $i$, $1 \leq i \leq r$, then $x^{q^{k}}=x, \forall x \in \zeta\left(F C_{32}\right)$ if and only if $y_{i}^{q^{k}}=1$, which holds if and only if $\left[D_{i}: F\right] \mid k$, for all $1 \leq i \leq r$. Hence the least number $t$ such that $32 \mid q^{k}-1$ or $32 \mid q^{k}+1$,

$$
t=\text { l.c.m. }\left\{\left[D_{i}: F\right] \mid 1 \leq i \leq r\right\} .
$$

Therefore all conjugacy classes of $C_{32}$ are $p$-regular and $m=32$. By observation we have following possibilities for $q$ :

(a) If $q \equiv 1 \bmod 32$, then $t=1$;

(b) If $q \equiv-1 \bmod 32$, then $t=2$;

(c) If $q \equiv 3,-5,11,-13 \bmod 32$, then $t=8$;

(d) If $q \equiv-3,5,-11,13 \bmod 32$, then $t=8$;

(e) If $q \equiv 7 \bmod 32$, then $t=4$;

(f) If $q \equiv-7 \bmod 32$, then $t=4$;

(g) If $q \equiv 15 \bmod 32$, then $t=2$;

(h) If $q \equiv-15 \bmod 32$, then $t=2$.

Now we will find $T$ and the number of $p$-regular $F$-conjugacy classes, denoted by $c$. By Lemma $4, \operatorname{dim}_{F}\left(\zeta\left(F C_{32}\right)\right)=32$, therefore $\sum_{i=1}^{r}\left[D_{i}\right.$ : $F]=32$. We have the following cases:

1. If $q \equiv 1 \bmod 32$, then $T=\{1\} \bmod 32$. Thus $p$-regular $F$-conjugacy classes are the conjugacy classes of $C_{32}$ and $c=32$. Hence $F C_{32} \cong F^{32}$.

2. If $q \equiv-1 \bmod 32$, then $T=\{1,-1\} \bmod 32$. Thus $p$-regular $F$ conjugacy classes are $\{1\},\left\{a^{16}\right\},\left\{a^{ \pm i}\right\}, 1 \leq i \leq 15$ and $c=17$. Hence $F C_{32} \cong F^{2} \oplus F_{2}^{15}$. 
3. If $q \equiv 3,-5,11,-13 \bmod 32$, then $T=\{1,3,9,11,17,19,25,27\} \bmod 32$.

Thus $p$-regular $F$-conjugacy classes are $\{1\},\left\{a, a^{3}, a^{9}, a^{11}, a^{17}, a^{19}, a^{25}, a^{27}\right\}$, $\left\{a^{2}, a^{6}, a^{18}, a^{22}\right\},\left\{a^{4}, a^{12}\right\},\left\{a^{5}, a^{7}, a^{13}, a^{15}, a^{21}, a^{23}, a^{29}, a^{31}\right\},\left\{a^{8}, a^{24}\right\}$, $\left\{a^{10}, a^{14}, a^{26}, a^{30}\right\},\left\{a^{16}\right\},\left\{a^{20}, a^{28}\right\}$ and $c=9$. Hence $F C_{32} \cong F_{8}^{2} \oplus$ $F_{4}^{2} \oplus F_{2}^{3} \oplus F^{2}$.

4. If $q \equiv-3,5,-11,13 \bmod 32$, then $T=\{1,5,9,13,17,21,25,29\} \bmod 32$.

Thus $p$-regular $F$-conjugacy classes are $\{1\},\left\{a, a^{5}, a^{9}, a^{13}, a^{17}, a^{21}, a^{25}, a^{29}\right\}$, $\left\{a^{2}, a^{10}, a^{18}, a^{26}\right\},\left\{a^{4}, a^{20}\right\},\left\{a^{3}, a^{7}, a^{11}, a^{15}, a^{19}, a^{23}, a^{27}, a^{31}\right\},\left\{a^{8}\right\}$, $\left\{a^{6}, a^{14}, a^{22}, a^{30}\right\},\left\{a^{16}\right\},\left\{a^{24}\right\},\left\{a^{12}, a^{28}\right\}$ and $c=10$. Hence $F C_{32} \cong$ $F_{8}^{2} \oplus F_{4}^{2} \oplus F_{2}^{2} \oplus F^{4}$.

5. If $q \equiv 7 \bmod 32$, then $T=\{1,7,17,23\} \bmod 32$. Thus, $p$-regular $F$ conjugacy classes are $\{1\},\left\{a, a^{7}, a^{17}, a^{23}\right\},\left\{a^{2}, a^{14}\right\},\left\{a^{3}, a^{5}, a^{19}, a^{21}\right\}$, $\left\{a^{4}, a^{28}\right\},\left\{a^{6}, a^{10}\right\},\left\{a^{8}, a^{24}\right\},\left\{a^{9}, a^{15}, a^{25}, a^{31}\right\},\left\{a^{11}, a^{13}, a^{27}, a^{29}\right\}$, $\left\{a^{12}, a^{20}\right\},\left\{a^{16}\right\},\left\{a^{18}, a^{30}\right\},\left\{a^{22}, a^{26}\right\}$ and $c=13$. Hence $F C_{32} \cong$ $F^{2} \oplus F_{4}^{4} \oplus F_{2}^{7}$.

6. If $q \equiv-7 \bmod 32$, then $T=\{1,9,17,25\} \bmod 32$. Thus, $p$-regular $F$ conjugacy classes are $\{1\},\left\{a, a^{9}, a^{17}, a^{25}\right\},\left\{a^{2}, a^{18}\right\},\left\{a^{3}, a^{11}, a^{19}, a^{27}\right\}$, $\left\{a^{4}\right\},\left\{a^{6}, a^{22}\right\},\left\{a^{5}, a^{13}, a^{21}, a^{29}\right\},\left\{a^{7}, a^{15}, a^{23}, a^{31}\right\},\left\{a^{8}\right\},\left\{a^{10}, a^{26}\right\}$, $\left\{a^{12}\right\},\left\{a^{16}\right\},\left\{a^{14}, a^{30}\right\},\left\{a^{20}\right\},\left\{a^{24}\right\},\left\{a^{28}\right\}$ and $c=16$. Hence $F C_{32} \cong F^{8} \oplus F_{2}^{4} \oplus F_{4}^{4}$.

7. If $q \equiv 15 \bmod 32$, then $T=\{1,15\} \bmod 32$. Thus, $p$-regular $F$-conjugacy classes are $\{1\},\left\{a, a^{15}\right\},\left\{a^{2}, a^{30}\right\},\left\{a^{3}, a^{13}\right\},\left\{a^{4}, a^{28}\right\},\left\{a^{5}, a^{11}\right\},\left\{a^{6}, a^{26}\right\}$, $\left\{a^{7}, a^{9}\right\},\left\{a^{8}, a^{24}\right\},\left\{a^{10}, a^{22}\right\},\left\{a^{12}, a^{20}\right\},\left\{a^{14}, a^{18}\right\},\left\{a^{17}, a^{31}\right\},\left\{a^{19}, a^{29}\right\}$, $\left\{a^{21}, a^{27}\right\},\left\{a^{23}, a^{25}\right\},\left\{a^{16}\right\}$ and $c=17$. Hence, $F C_{32} \cong F^{2} \oplus F_{2}^{15}$.

8. If $q \equiv-15 \bmod 32$, then $T=\{1,17\} \bmod 32$. Thus, $p$-regular $F$ conjugacy classes are $\{1\},\left\{a, a^{17}\right\},\left\{a^{2}\right\},\left\{a^{30}\right\},\left\{a^{3}, a^{19}\right\},\left\{a^{4}\right\},\left\{a^{28}\right\}$, $\left\{a^{5}, a^{21}\right\},\left\{a^{6}\right\},\left\{a^{26}\right\},\left\{a^{7}, a^{23}\right\},\left\{a^{8}\right\},\left\{a^{24}\right\},\left\{a^{9}, a^{25}\right\},\left\{a^{10}\right\},\left\{a^{22}\right\}$, $\left\{a^{11}, a^{27}\right\},\left\{a^{13}, a^{29}\right\},\left\{a^{15}, a^{31}\right\},\left\{a^{12}\right\},\left\{a^{20}\right\},\left\{a^{16}\right\},\left\{a^{14}\right\},\left\{a^{18}\right\}$ and $c=24$. Hence, $F C_{32} \cong F^{16} \oplus F_{2}^{8}$. Thus our result follows.

Theorem 2. Let $F$ be a finite field of characteristic $p>0$ having $q=p^{n}$ elements and $G \cong C_{16} \times C_{2}$.

1. If $p=2$. Then, $U\left(F\left[C_{16} \times C_{2}\right]\right) \cong C_{16}^{n} \times C_{8}^{n} \times C_{4}^{2 n} \times C_{2}^{20 n} \times C_{2^{n}-1}$. 
2. If $p \neq 2$. Then,

$U\left(F\left[C_{16} \times C_{2}\right]\right) \cong \begin{cases}C_{p^{n}-1}^{32}, & \text { if } q \equiv 1 \bmod 16 ; \\ C_{p^{n}-1}^{4} \times C_{p^{2 n}-1}^{14}, & \text { if } q \equiv-1 \bmod 16 ; \\ C_{p^{n}-1}^{4} \times C_{p^{2 n}-1}^{6} \times C_{p^{4 n}-1}^{4}, & \text { if } q \equiv 3,-5 \bmod 16 ; \\ C_{p^{n}-1}^{8} \times C_{p^{2 n}-1}^{4} \times C_{p^{4 n}-1}^{4}, & \text { if } q \equiv-3,5 \bmod 16 ; \\ C_{p^{n}-1}^{4} \times C_{p^{2 n}-1}^{14}, & \text { if } q \equiv 7 \bmod 16 ; \\ C_{p^{n}-1}^{16} \times C_{p^{2 n}-1}^{8}, & \text { if } q \equiv-7 \bmod 16 .\end{cases}$

Proof. The presentation of $G \cong C_{16} \times C_{2}$ is given by

$$
C_{16} \times C_{2}=<a, b \mid a^{16}=b^{2}=1, a b=b a>.
$$

1. If $p=2$, then $F G$ is non-semisimple and $|F|=q=2^{n}$. It is well known that $U(F G) \cong V(F G) \times F^{*}$ and $|V(F G)|=2^{31 n}$ as $\operatorname{dim}_{F} J(F G)=31$. Obviously exponent of $V(F G)$ is 16. Suppose $V(F G) \cong C_{16}^{l_{1}} \times C_{8}^{l_{2}} \times C_{4}^{l_{3}} \times C_{2}^{l_{4}}$ such that $2^{31 n}=16^{l_{1}} \times 8^{l_{2}} \times 4^{l_{3}} \times 2^{l_{4}}$. Now we will compute $l_{1}, l_{2}, l_{3}$ and $l_{4}$. Set $W_{1}=\left\{\gamma_{1} \in \omega(G): \gamma_{1}^{2}=\right.$ 0 and there exists $\beta \in \omega(G)$, such that $\left.\gamma_{1}=\beta^{8}\right\}, W_{2}=\left\{\gamma_{2} \in \omega(G)\right.$ : $\gamma_{2}^{2}=0$ and there exists $\beta \in \omega(G)$, such that $\left.\gamma_{2}=\beta^{4}\right\}$ and $W_{3}=$ $\left\{\gamma_{3} \in \omega(G): \gamma_{3}^{2}=0\right.$ and there exists $\beta \in \omega(G)$, such that $\left.\gamma_{3}=\beta^{2}\right\}$. Now if $\gamma=\sum_{j=0}^{1} \sum_{i=0}^{15} \alpha_{16 j+i} a^{i} b^{j} \in \omega(G)$, then $\sum_{i=0}^{15} \alpha_{2 i+j}=0$, for $j=0,1$. Also $\gamma^{2}=\sum_{j=0}^{7} \sum_{i=0}^{3} \alpha_{8 i+j}^{2} a^{2 j}, \gamma^{4}=\sum_{j=0}^{3} \sum_{i=0}^{7} \alpha_{4 i+j}^{4} a^{4 j}$ and $\gamma^{8}=\sum_{j=0}^{1} \sum_{i=0}^{15} \alpha_{2 i+j}^{8} a^{8 j}$. Let $\beta=\sum_{j=0}^{1} \sum_{i=0}^{15} \beta_{16 j+i} a^{i} b^{j}$, such that $\gamma_{1}=\beta^{8}$. Now applying condition $\gamma_{1}^{2}=0$ and by direct computation we have $\alpha_{i}=0$, for all $i \neq 0,8$ and $\alpha_{0}=\alpha_{8}$. Thus $W_{1}=\left\{\alpha_{0}\left(1+a^{8}\right), \alpha_{0} \in F\right\},\left|W_{1}\right|=2^{n}$ and $l_{1}=n$. Similarly, applying the conditions $\gamma_{2}=\beta^{4}$ and $\gamma_{2}^{2}=0$, we have $\alpha_{i}=0$, for all $i \neq 0,4$ and $\alpha_{0}=\alpha_{4}$. Thus $W_{2}=\left\{\alpha_{0}\left(1+a^{4}\right), \alpha_{0} \in F\right\},\left|W_{2}\right|=2^{n}$ and $l_{2}=n$. Again, applying the conditions $\gamma_{3}=\beta^{8}$ and $\gamma_{3}^{2}=0$. We have $\alpha_{i}=0$, for all $i \neq 0,2,8,10$ and $\alpha_{0}=\alpha_{8}, \alpha_{2}=\alpha_{10}$. Thus $W_{3}=\left\{\left(\alpha_{0}+\alpha_{2} a^{2}\right)\left(1+a^{8}\right), \alpha_{0}, \alpha_{2} \in F\right\}, l_{3}=2 n$ and $l_{4}=20 n$. Hence $V(F G) \cong C_{16}^{n} \times C_{8}^{n} \times C_{4}^{2 n} \times C_{2}^{20 n}$ and hence the result.

2. If $p \neq 2$, then $|F|=p^{n}$. Using the similar arguments as in Theorem $1, F\left[C_{16} \times C_{2}\right]$ is semisimple and we have $m=16, \sum_{i=1}^{r}\left[D_{i}: F\right]=32$. By observation we have following possibilities for $q$ : 
(a) If $q \equiv 1 \bmod 16$, then $t=1$;

(b) If $q \equiv-1 \bmod 16$, then $t=2$;

(c) If $q \equiv 3,-5 \bmod 16$, then $t=4$;

(d) If $q \equiv-3,5 \bmod 16$, then $t=4$;

(e) If $q \equiv 7 \bmod 16$, then $t=2$;

(f) If $q \equiv-7 \bmod 16$, then $t=2$.

Hence we have the following cases:

1. If $q \equiv 1 \bmod 16$, then $T=\{1\} \bmod 16$. Thus, $p$-regular $F$-conjugacy classes are the conjugacy classes of $C_{16} \times C_{2}$ and $c=32$. Hence $F\left[C_{16} \times\right.$ $\left.C_{2}\right] \cong F^{32}$.

2. If $q \equiv-1 \bmod 16$, then $T=\{1,-1\} \bmod 16$. Thus, $p$-regular $F$ conjugacy classes are $\{1\},\{b\},\left\{a^{8}\right\},\left\{a^{ \pm i}\right\}$, where $1 \leq i \leq 7,\left\{a^{8} b\right\}$, $\left\{a^{j} b, a^{-j} b\right\}$, where $1 \leq j \leq 7$ and $c=18$. Hence $F\left[C_{16} \times C_{2}\right] \cong F^{4} \oplus$ $F_{2}^{14}$.

3. If $q \equiv 3,-5 \bmod 16$, then $T=\{1,3,9,11\} \bmod 16$. Thus, $p$-regular $F$-conjugacy classes are $\{1\},\{b\},\left\{a, a^{3}, a^{-7}, a^{-5}\right\}$, $\left\{a^{-1}, a^{-3}, a^{5}, a^{7}\right\},\left\{a^{2}, a^{6}\right\},\left\{a^{-2}, a^{-6}\right\},\left\{a^{ \pm 4}\right\},\left\{a^{8}\right\},\left\{a b, a^{3} b\right.$, $\left.a^{-7} b, a^{-5} b\right\},\left\{a^{-1} b, a^{-3} b, a^{5} b, a^{7} b\right\},\left\{a^{2} b, a^{6} b\right\},\left\{a^{-2} b, a^{-6} b\right\},\left\{a^{ \pm 4} b\right\}$, $\left\{a^{8} b\right\}$ and $c=14$. Hence $F\left[C_{16} \times C_{2}\right] \cong F_{2}^{6} \oplus F_{4}^{4} \oplus F^{4}$.

4. If $q \equiv-3,5 \bmod 16$, then $T=\{1,5,9,13\} \bmod 16$. Thus, $p$-regular $F$-conjugacy classes are $\{1\},\{b\},\left\{a, a^{5}, a^{-3}, a^{-7}\right\}$, $\left\{a^{-1}, a^{-5}, a^{3}, a^{7}\right\},\left\{a^{2}, a^{-6}\right\},\left\{a^{-2}, a^{6}\right\},\left\{a^{4}\right\},\left\{a^{-4}\right\},\left\{a^{8}\right\}$, $\left\{a b, a^{5} b, a^{-3} b, a^{-7} b\right\},\left\{a^{-1} b, a^{-5} b, a^{3} b, a^{7} b\right\},\left\{a^{2} b, a^{-6} b\right\},\left\{a^{-2} b, a^{6} b\right\}$, $\left\{a^{4} b\right\},\left\{a^{-4} b\right\},\left\{a^{8} b\right\}$ and $c=16$. Hence $F\left[C_{16} \times C_{2}\right] \cong F_{2}^{4} \oplus F_{4}^{4} \oplus F^{8}$.

5. If $q \equiv 7 \bmod 16$, then $T=\{1,7\} \bmod 16$. Thus, $p$-regular $F$-conjugacy classes are $\{1\},\{b\},\left\{a, a^{7}\right\},\left\{a^{3}, a^{5}\right\},\left\{a^{-1}, a^{-7}\right\},\left\{a^{-3}, a^{-5}\right\},\left\{a^{ \pm 2}\right\}$, $\left\{a^{ \pm 6}\right\},\left\{a^{ \pm 4}\right\},\left\{a^{8}\right\},\left\{a b, a^{7} b\right\},\left\{a^{3} b, a^{5} b\right\},\left\{a^{-1} b, a^{-7} b\right\},\left\{a^{-3} b, a^{-5} b\right\}$, $\left\{a^{ \pm 2} b\right\},\left\{a^{ \pm 6} b\right\},\left\{a^{ \pm 4} b\right\},\left\{a^{8} b\right\}$ and $c=18$. Hence $F\left[C_{16} \times C_{2}\right] \cong F_{2}^{14} \oplus$ $F^{4}$.

6. If $q \equiv-7 \bmod 16$, then $T=\{1,9\} \bmod 16$. Thus, $p$-regular $F$-conjugacy classes are $\{1\},\{b\},\left\{a, a^{-7}\right\},\left\{a^{3}, a^{-5}\right\},\left\{a^{-1}, a^{7}\right\},\left\{a^{-3}, a^{5}\right\},\left\{a^{2}\right\}$, $\left\{a^{-2}\right\},\left\{a^{6}\right\},\left\{a^{-6}\right\},\left\{a^{4}\right\},\left\{a^{-4}\right\},\left\{a^{8}\right\},-\left\{a b, a^{-7} b\right\},\left\{a^{3} b, a^{-5} b\right\},\left\{a^{-1} b, a^{7} b\right\}$, $\left\{a^{-3} b, a^{5} b\right\},\left\{a^{2} b\right\},\left\{a^{-2} b\right\},\left\{a^{6} b\right\},\left\{a^{-6} b\right\},\left\{a^{4} b\right\},\left\{a^{-4} b\right\},\left\{a^{8} b\right\}$ and $c=24$. Hence $F\left[C_{16} \times C_{2}\right] \cong F_{2}^{8} \oplus F^{16}$. Thus we have the result. 
Theorem 3. Let $F$ be a finite field of characteristic $p>0$ having $q=p^{n}$ elements and $G \cong C_{8} \times C_{4}$.

1. If $p=2$. Then,

$$
U\left(F\left[C_{8} \times C_{4}\right]\right) \cong C_{8}^{n} \times C_{4}^{5 n} \times C_{2}^{18 n} \times C_{2^{n}-1} .
$$

2. If $p \neq 2$. Then,

$$
U\left(F\left[C_{8} \times C_{4}\right]\right) \cong\left\{\begin{array}{lll}
C_{p^{n}-1}^{32}, & \text { if } q \equiv 1 \bmod 8 \\
C_{p^{n}-1}^{4} \times C_{p^{2 n}-1}^{14}, & \text { if } q \equiv-1 \bmod 8 \\
C_{p^{n}-1}^{4} \times C_{p^{2 n}-1}^{14}, & \text { if } q \equiv 3 \bmod 8 \\
C_{p^{n}-1}^{16} \times C_{p^{2 n}-1}^{8}, & \text { if } q \equiv-3 \bmod 8
\end{array}\right.
$$

Proof. The presentation of $G \cong C_{8} \times C_{4}$ is given by

$$
C_{8} \times C_{4}=<a, b \mid a^{8}=b^{4}=1, a b=b a>.
$$

1. If $p=2$, then $F G$ is non-semisimple and $|F|=q=2^{n}$. It is well known that $U(F G) \cong V(F G) \times F^{*}$ and $|V(F G)|=2^{31 n}$ as $\operatorname{dim}_{F} J(F G)=31$. Obviously exponent of $V(F G)$ is 8. Suppose $V(F G) \cong C_{8}^{l_{1}} \times C_{4}^{l_{2}} \times C_{2}^{l_{3}}$ such that $2^{31 n}=8^{l_{1}} \times 4^{l_{2}} \times 2^{l_{3}}$. Now we will compute $l_{1}, l_{2}$ and $l_{3}$. Set $W_{1}=\left\{\alpha \in \omega(G): \alpha^{2}=0\right.$ and there exists $\beta \in$ $\omega(G)$, such that $\left.\alpha=\beta^{4}\right\}, W_{2}=\left\{\gamma \in \omega(G): \gamma^{2}=0\right.$ and there exists $\beta \in$ $\omega(G)$, such that $\left.\gamma=\beta^{2}\right\}$.

If $\alpha=\sum_{j=0}^{3} \sum_{i=0}^{7} \alpha_{8 j+i} a^{i} b^{j} \in \omega(G)$, then $\sum_{i=0}^{7} \alpha_{4 i+j}=0$, for $j=$ $0,1,2,3$. Let $\beta=\sum_{j=0}^{3} \sum_{i=0}^{7} \beta_{8 j+i} a^{i} b^{j}$ such that $\alpha=\beta^{4}$. Now applying condition $\alpha^{2}=0, \alpha=\beta^{4}$ and by direct computation, we have $\alpha_{i}=0$, for all $i \neq 0,4$ and $\alpha_{0}=\alpha_{4}$. Thus $W_{1}=\left\{\alpha_{0}\left(1+a^{4}\right), \alpha_{0} \in F\right\}$. Therefore $\left|W_{1}\right|=2^{n}$ and $l_{1}=n$. Similarly, applying the conditions $\gamma=\beta^{2}, \gamma^{2}=0$ and by direct computation, we have $\left|W_{2}\right|=2^{5 n}$, $l_{2}=5 n$ and $l_{3}=18 n$. Hence $V(F G) \cong C_{8}^{n} \times C_{4}^{5 n} \times C_{2}^{18 n}$ and hence the result.

2. If $p \neq 2$, then $|F|=p^{n}$. Using the similar arguments as in Theorem $1, F\left[C_{8} \times C_{4}\right]$ is semisimple and we have $m=8, \sum_{i=1}^{r}\left[D_{i}: F\right]=32$. By observation we have following possibilities for $q$ : 
(a) If $q \equiv 1 \bmod 8$, then $t=1$;

(b) If $q \equiv-1 \bmod 8$, then $t=2$;

(c) If $q \equiv 3 \bmod 8$, then $t=2$;

(d) If $q \equiv-3 \bmod 8$, then $t=2$.

Hence we have the following cases: -

1. If $q \equiv 1 \bmod 8$, then $T=\{1\} \bmod 8$. Thus, $p$-regular $F$-conjugacy classes are the conjugacy classes of $C_{8} \times C_{4}$ and $c=32$. Hence $F\left[C_{8} \times\right.$ $\left.C_{4}\right] \cong F^{32}$.

2. If $q \equiv-1 \bmod 8$, then $T=\{1,-1\} \bmod 8$. Thus, $p$-regular $F$-conjugacy classes are $\{1\},\left\{b^{2}\right\},\left\{b, b^{3}\right\},\left\{a^{ \pm 1}\right\},\left\{a^{ \pm 2}\right\},\left\{a^{ \pm 3}\right\},\left\{a^{4}\right\},\left\{a b, a^{-1} b^{3}\right\}$, $\left\{a^{2} b, a^{-2} b^{3}\right\},\left\{a^{3} b, a^{-3} b^{3}\right\},\left\{a^{4} b, a^{4} b^{3}\right\},\left\{a^{-3} b, a^{3} b^{3}\right\},\left\{a^{-2} b, a^{2} b^{3}\right\},\left\{a^{-1} b, a b^{3}\right\}$, $\left\{a b^{2}, a^{-1} b^{2}\right\},\left\{a^{-2} b^{2}, a^{2} b^{2}\right\},\left\{a^{3} b^{2}, a^{-3} b^{2}\right\},\left\{a^{4} b^{2}\right\}$ and $c=18$. Hence $F\left[C_{8} \times C_{4}\right] \cong F^{4} \oplus F_{2}^{14}$.

3. If $q \equiv 3 \bmod 8$, then $T=\{1,3\} \bmod 8$. Thus, $p$-regular $F$-conjugacy classes are $\{1\},\left\{b^{2}\right\},\left\{b, b^{3}\right\},\left\{a, a^{3}\right\},\left\{a^{2}, a^{-2}\right\},\left\{a^{-1}, a^{-3}\right\},\left\{a^{4}\right\}$, $\left\{a b, a^{3} b^{3}\right\},\left\{a^{2} b, a^{-2} b^{3}\right\},\left\{a^{-1} b, a^{-3} b^{3}\right\},\left\{a^{4} b, a^{4} b^{3}\right\},\left\{a b^{3}, a^{3} b\right\},\left\{a^{2} b^{3}, a^{-2} b\right\}$, $\left\{a^{-1} b^{3}, a^{-3} b\right\},\left\{a b^{2}, a^{3} b^{2}\right\},\left\{a^{2} b^{2}, a^{-2} b^{2}\right\},\left\{a^{-1} b^{2}, a^{-3} b^{2}\right\},\left\{a^{4} b^{2}\right\}$ and $c=18$. Hence $F\left[C_{8} \times C_{4}\right] \cong F^{4} \oplus F_{2}^{14}$.

4. If $q \equiv-3 \bmod 8$, then $T=\{1,5\} \bmod 8$. Thus, $p$-regular $F$-conjugacy classes are $\{1\},\{b\},\left\{b^{2}\right\},\left\{b^{3}\right\},\left\{a, a^{-3}\right\},\left\{a^{2}\right\},\left\{a^{-2}\right\},\left\{a^{-1}, a^{3}\right\},\left\{a^{4}\right\}$, $\left\{a b, a^{-3} b\right\},\left\{a^{2} b\right\},\left\{a^{-2} b\right\},\left\{a^{-1} b, a^{3} b\right\},\left\{a^{4} b\right\},\left\{a b^{2}, a^{-3} b^{2}\right\},\left\{a^{2} b^{2}\right\}$, $\left\{a^{-2} b^{2}\right\},\left\{a^{-1} b^{2}, a^{3} b^{2}\right\},\left\{a^{4} b^{2}\right\},\left\{a b^{3}, a^{-3} b^{3}\right\},\left\{a^{2} b^{3}\right\},\left\{a^{-2} b^{3}\right\},\left\{a^{-1} b^{3}, a^{3} b^{3}\right\}$, $\left\{a^{4} b^{3}\right\}$ and $c=24$. Hence $F\left[C_{8} \times C_{4}\right] \cong F^{16} \oplus F_{2}^{8}$.

Thus we have the result.

Theorem 4. Let $F$ be a finite field of characteristic $p>0$ having $q=p^{n}$ elements and $G \cong C_{8} \times C_{2} \times C_{2}$.

1. If $p=2$. Then,

$$
U\left(F\left[C_{8} \times C_{2} \times C_{2}\right]\right) \cong C_{8}^{n} \times C_{4}^{n} \times C_{2}^{26 n} \times C_{2^{n}-1} .
$$

2. If $p \neq 2$. Then, 


$$
U\left(F\left[C_{8} \times C_{2} \times C_{2}\right]\right) \cong\left\{\begin{array}{lll}
C_{p^{n}-1}^{32}, & \text { if } q \equiv 1 \bmod 8 \\
C_{p^{n}-1}^{8} \times C_{p^{2 n}-1}^{12}, & \text { if } q \equiv-1 \bmod 8 ; \\
C_{p^{n}-1}^{8} \times C_{p^{2 n}-1}^{12}, & \text { if } q \equiv 3 \bmod 8 ; \\
C_{p^{n}-1}^{16} \times C_{p^{2 n}-1}^{8}, & \text { if } q \equiv-3 \bmod 8
\end{array}\right.
$$

Proof. The presentation of $G \cong C_{8} \times C_{2} \times C_{2}$ is given by

$C_{8} \times C_{2} \times C_{2}=<a, b, c \mid a^{8}=b^{2}=c^{2}=1, a b=b a, b c=c b, a c=c a>$.

1. If $p=2$, then $F G$ is non-semisimple and $|F|=q=2^{n}$. It is well known that $U(F G) \cong V(F G) \times F^{*}$ and $|V(F G)|=2^{31 n}$ as $\operatorname{dim}_{F} J(F G)=31$. Obviously exponent of $V(F G)$ is 8. Suppose $V(F G) \cong C_{8}^{l_{1}} \times C_{4}^{l_{2}} \times C_{2}^{l_{3}}$ such that $2^{31 n}=8^{l_{1}} \times 4^{l_{2}} \times 2^{l_{3}}$. Now we will compute $l_{1}, l_{2}$ and $l_{3}$. Set $W_{1}=\left\{\alpha \in \omega(G): \alpha^{2}=0\right.$ and there exists $\beta \in$ $\omega(G)$, such that $\left.\alpha=\beta^{4}\right\}, W_{2}=\left\{\gamma \in \omega(G): \gamma^{2}=0\right.$ and there exists $\beta \in$ $\omega(G)$, such that $\left.\gamma=\beta^{2}\right\}$.

Let $\alpha=\sum_{k=0}^{1} \sum_{j=0}^{1} \sum_{i=0}^{7} \alpha_{8}(j+2 k)+i a^{i} b^{j} c^{k} \in \omega(G)$ and $\beta=\sum_{k=0}^{1} \sum_{j=0}^{1} \sum_{i=0}^{7} \beta_{8(j+2 k)+i} a^{i} b^{j} c^{k}$ such that $\alpha=\beta^{4}$. Now applying the conditions $\alpha^{2}=0, \alpha=\beta^{4}$ and by direct computation, we have $\alpha_{i}=0$, for all $i \neq 0,4$ and $\alpha_{0}=\alpha_{4}$. Thus $W_{1}=\left\{\alpha_{0}\left(1+a^{4}\right), \alpha_{0} \in F\right\}$. Therefore $\left|W_{1}\right|=2^{n}$ and $l_{1}=n$. Similarly, applying the conditions $\gamma=\beta^{2}, \gamma^{2}=0$ and by direct computation, we have $\alpha_{i}=0$, for all $i \neq 0,2$ and $\alpha_{0}=\alpha_{2}$. Thus $W_{2}=\left\{\alpha_{0}\left(1+a^{2}\right), \alpha_{0} \in F\right\}$. Therefore $\left|W_{2}\right|=2^{n}, l_{2}=n$ and $l_{3}=26 n$. Hence $V(F G) \cong C_{8}^{n} \times C_{4}^{n} \times C_{2}^{26 n}$ and hence the result follows.

2. If $p \neq 2$, then $|F|=p^{n}$. Using the similar arguments as in Theorem $1, F\left[C_{8} \times C_{2} \times C_{2}\right]$ is semisimple and $m=8, \sum_{i=1}^{r}\left[D_{i}: F\right]=32$. Here the number of $p$-regular $F$-conjugacy classes, denoted by $w$. By observation we have following possibilities for $q$ :

(a) If $q \equiv 1 \bmod 8$, then $t=1$;

(b) If $q \equiv-1 \bmod 8$, then $t=2$;

(c) If $q \equiv 3 \bmod 8$, then $t=2$;

(d) If $q \equiv-3 \bmod 8$, then $t=2$.

Now we have the cases: 
1. If $q \equiv 1 \bmod 8$, then $T=\{1\} \bmod 8$. Thus, $p$-regular $F$-conjugacy classes are the conjugacy classes of $C_{8} \times C_{2} \times C_{2}$ and $w=32$. Hence $F\left[C_{8} \times C_{2} \times C_{2}\right] \cong F^{32}$.

2. If $q \equiv-1 \bmod 8$, then $T=\{1,7\} \bmod 8$. Thus, $p$-regular $F$-conjugacy classes are $\{1\},\left\{a, a^{7}\right\},\left\{a^{2}, a^{6}\right\},\left\{a^{3}, a^{5}\right\},\left\{a^{4}\right\},\{b\},\{c\},\left\{a b, a^{7} b\right\}$, $\left\{a^{2} b, a^{6} b\right\},\left\{a^{3} b, a^{5} b\right\},\left\{a^{4} b\right\},\left\{a c, a^{7} c\right\},\left\{a^{2} c, a^{6} c\right\},\left\{a^{3} c, a^{5} c\right\},\left\{a^{4} c\right\}$, $\{b c\},\left\{a b c, a^{7} b c\right\},\left\{a^{2} b c, a^{6} b c\right\},\left\{a^{3} b c, a^{5} b c\right\},\left\{a^{4} b c\right\}$ and $w=20$. Hence $F\left[C_{8} \times C_{2} \times C_{2}\right] \cong F^{8} \oplus F_{2}^{12}$.

3. If $q \equiv 3 \bmod 8$, then $T=\{1,3\} \bmod 8$. Thus, $p$-regular $F$-conjugacy classes are $\{1\},\left\{a, a^{3}\right\},\left\{a^{2}, a^{6}\right\},\left\{a^{5}, a^{7}\right\},\left\{a^{4}\right\},\{b\},\{c\},\left\{a b, a^{3} b\right\}$, $\left\{a^{2} b, a^{6} b\right\},\left\{a^{5} b, a^{7} b\right\},\left\{a^{4} b\right\},\left\{a c, a^{3} c\right\},\left\{a^{2} c, a^{6} c\right\},\left\{a^{5} c, a^{7} c\right\},\left\{a^{4} c\right\}$, $\{b c\},\left\{a b c, a^{3} b c\right\},\left\{a^{2} b c, a^{6} b c\right\},\left\{a^{5} b c, a^{7} b c\right\},\left\{a^{4} b c\right\}$ and $w=20$. Hence $F\left[C_{8} \times C_{2} \times C_{2}\right] \cong F^{8} \oplus F_{2}^{12}$.

4. If $q \equiv-3 \bmod 8$, then $T=\{1,5\} \bmod 8$. Thus, $p$-regular $F$-conjugacy classes are $\{1\},\left\{a, a^{5}\right\},\left\{a^{2}\right\},\left\{a^{6}\right\},\left\{a^{3}, a^{7}\right\},\left\{a^{4}\right\},\{b\},\{c\},\left\{a b, a^{5} b\right\}$, $\left\{a^{2} b\right\},\left\{a^{6} b\right\},\left\{a^{3} b, a^{7} b\right\},\left\{a^{4} b\right\},\left\{a c, a^{5} c\right\},\left\{a^{2} c\right\},\left\{a^{6} c\right\},\left\{a^{3} c, a^{7} c\right\}$, $\left\{a^{4} c\right\},\{b c\},\left\{a b c, a^{5} b c\right\},\left\{a^{2} b c\right\},\left\{a^{6} b c\right\},\left\{a^{3} b c, a^{7} b c\right\},\left\{a^{4} b c\right\}$ and $w=24$. Hence $F\left[C_{8} \times C_{2} \times C_{2}\right] \cong F^{16} \oplus F_{2}^{8}$.

Thus we have the result.

Theorem 5. Let $F$ be a finite field of characteristic $p>0$ having $q=p^{n}$ elements and $G \cong C_{4}^{2} \times C_{2}$.

1. If $p=2$. Then,

$$
U\left(F\left[C_{4}^{2} \times C_{2}\right]\right) \cong C_{4}^{3 n} \times C_{2}^{25 n} \times C_{2^{n}-1} .
$$

2. If $p \neq 2$. Then,

$$
U\left(F\left[C_{4}^{2} \times C_{2}\right]\right) \cong\left\{\begin{array}{lll}
C_{p^{n}-1}^{32}, & \text { if } & q \equiv 1 \bmod 4 \\
C_{p^{n}-1}^{8} \times C_{p^{2 n}-1}^{12}, & \text { if } & q \equiv-1 \bmod 4 .
\end{array}\right.
$$


Proof. The presentation of $G \cong C_{4}^{2} \times C_{2}$ is given by

$$
C_{4}^{2} \times C_{2}=<a, b, c \mid a^{4}=b^{4}=c^{2}=1, a b=b a, b c=c b, a c=c a>.
$$

1. If $p=2$, then $F G$ is non-semisimple and $|F|=q=2^{n}$. It is well known that $U(F G) \cong V(F G) \times F^{*}$ and $|V(F G)|=2^{31 n}$ as $\operatorname{dim}_{F} J(F G)=31$. Obviously exponent of $V(F G)$ is 4. Suppose $V(F G) \cong C_{4}^{l_{1}} \times C_{2}^{l_{2}}$ such that $2^{31 n}=4^{l_{1}} \times 2^{l_{2}}$. Now we will compute $l_{1}$ and $l_{2}$. Set $W=\left\{\alpha \in \omega(G): \alpha^{2}=0\right.$ and there exists $\beta \in$ $\omega(G)$, such that $\left.\alpha=\beta^{2}\right\}$. If $\alpha=\sum_{k=0}^{1} \sum_{j=0}^{3} \sum_{i=0}^{3} \alpha_{4(j+4 k)+i} a^{i} b^{j} c^{k} \in$ $\omega(G)$, then $\sum_{i=0}^{3} \alpha_{2(j+2 k)+i}=0$, for $j=0,1,2,3$ and $k=0,1$. Let $\beta=\sum_{k=0}^{1} \sum_{j=0}^{3} \sum_{i=0}^{3} \beta_{4(j+4 k)+i} a^{i} b^{j} c^{k}$ such that $\alpha=\beta^{2}$. Now applying the conditions $\alpha^{2}=0, \alpha=\beta^{2}$ and by direct computation, we have $\alpha_{i}=0$, for all $i \neq 0,2,8,10$ and $\alpha_{0}=\alpha_{2}$. Thus $W=$ $\left\{\alpha_{0}\left(1+a^{2}\right)+\left(\alpha_{8}+\alpha_{10} a^{2}\right) b^{2}, \alpha_{0}, \alpha_{8}, \alpha_{10} \in F\right\}$. Therefore $|W|=2^{3 n}$, $l_{1}=3 n$ and $l_{2}=25 n$. Hence $V(F G) \cong C_{4}^{3 n} \times C_{2}^{25 n}$ and the result follows.

2. If $p \neq 2$, then $|F|=p^{n}$. Using the similar arguments as in Theorem $1, F\left[C_{4}^{2} \times C_{2}\right]$ is semisimple and $m=4, \sum_{i=1}^{r}\left[D_{i}: F\right]=32$. By observation we have following possibilities for $q$ :

(a) If $q \equiv 1 \bmod 4$, then $t=1$;

(b) If $q \equiv-1 \bmod 4$, then $t=2$.

Now we have the cases:

1. If $q \equiv 1 \bmod 4$, then $T=\{1\} \bmod 4$. Thus, $p$-regular $F$-conjugacy classes are the conjugacy classes of $C_{4}^{2} \times C_{2}$ and $w=32$. Hence $F\left[C_{4}^{2} \times\right.$ $\left.C_{2}\right] \cong F^{32}$.

2. If $q \equiv-1 \bmod 4$, then $T=\{1,3\} \bmod 4$. Thus, $p$-regular $F$-conjugacy classes are $\{1\},\left\{a, a^{3}\right\},\left\{a^{2}\right\},\left\{b, b^{3}\right\},\left\{b^{2}\right\},\{c\},\left\{a b, a^{3} b^{3}\right\},\left\{a b^{2}, a^{3} b^{2}\right\}$, $\left\{a b^{3}, a^{3} b\right\},\left\{a^{2} b, a^{2} b^{3}\right\},\left\{a^{2} b^{2}\right\},\left\{b c, b^{3} c\right\},\left\{b^{2} c\right\},\left\{a b c, a^{3} b^{3} c\right\},\left\{a b^{2} c, a^{3} b^{2} c\right\}$, $\left\{a b^{3} c, a^{3} b c\right\},\left\{a^{2} b c, a^{2} b^{3} c\right\},\left\{a^{2} b^{2} c\right\},\left\{a c, a^{3} c\right\},\left\{a^{2} c\right\}$ and $w=20$. Hence $F\left[C_{4}^{2} \times C_{2}\right] \cong F^{8} \oplus F_{2}^{12}$. Thus we have the result.

Theorem 6. Let $F$ be a finite field of characteristic $p>0$ having $q=p^{n}$ elements and $G \cong C_{4} \times C_{2}^{3}$. 
1. If $p=2$. Then,

$$
U\left(F\left[C_{4} \times C_{2}^{3}\right]\right) \cong C_{4}^{n} \times C_{2}^{29 n} \times C_{2^{n}-1} .
$$

2. If $p \neq 2$. Then,

$$
U\left(F\left[C_{4} \times C_{2}^{3}\right]\right) \cong \begin{cases}C_{p^{n}-1}^{32}, & \text { if } \quad q \equiv 1 \bmod 4 \\ C_{p^{n}-1}^{16} \times C_{p^{2 n}-1}^{8}, & \text { if } \quad q \equiv-1 \bmod 4 .\end{cases}
$$

Proof. The presentation of $G \cong C_{4} \times C_{2}^{3}$ is given by

$C_{4} \times C_{2}^{3}=<a, b, c, d \mid a^{4}=b^{2}=c^{2}=d^{2}=1, a b=b a, b c=c b, d c=c d, a d=d a>$.

1. If $p=2$, then $F G$ is non-semisimple and $|F|=q=2^{n}$. It is well known that $U(F G) \cong V(F G) \times F^{*}$ and $|V(F G)|=2^{31 n}$ as $\operatorname{dim}_{F} J(F G)=31$. Obviously exponent of $V(F G)$ is 4. Suppose $V(F G) \cong C_{4}^{l_{1}} \times C_{2}^{l_{2}}$ such that $2^{31 n}=4^{l_{1}} \times 2^{l_{2}}$. Now we will compute $l_{1}$ and $l_{2}$. Set

$W=\left\{\alpha \in \omega(G): \alpha^{2}=0\right.$ and there exists $\beta \in \omega(G)$, such that $\left.\alpha=\beta^{2}\right\}$.

Let $\alpha=\sum_{s=0}^{1} \sum_{k=0}^{1} \sum_{j=0}^{1} \sum_{i=0}^{3} \alpha_{4(j+2(k+2 s))+i} a^{i} b^{j} c^{k} d^{s} \in \omega(G)$ and $\beta=\sum_{s=0}^{1} \sum_{k=0}^{1} \sum_{j=0}^{1} \sum_{i=0}^{3} \beta_{4(j+2(k+2 s))+i} a^{i} b^{j} c^{k} d^{s}$ such that $\alpha=\beta^{2}$. Now applying the conditions $\alpha^{2}=0, \alpha=\beta^{2}$ and by direct computation, we have $\alpha_{i}=0$, for all $i \neq 0,2$ and $\alpha_{0}=\alpha_{2}$. Thus $W=\left\{\alpha_{0}\left(1+a^{2}\right), \alpha_{0} \in F\right\}$. Therefore $|W|=2^{n}, l_{1}=n$ and $l_{2}=29 n$. Hence $V(F G) \cong C_{4}^{n} \times C_{2}^{29 n}$ and the result follows.

2. If $p \neq 2$, then $|F|=p^{n}$. Using the similar arguments as in Theorem $1, F\left[C_{4} \times C_{2}^{3}\right]$ is semisimple and $m=4, \sum_{i=1}^{r}\left[D_{i}: F\right]=32$. By observation we have following possibilities for $q$ :

(a) If $q \equiv 1 \bmod 4$, then $t=1$;

(b) If $q \equiv-1 \bmod 4$, then $t=2$.

Now have the following cases:

1. If $q \equiv 1 \bmod 4$, then $T=\{1\} \bmod 4$. Thus, $p$-regular $F$-conjugacy classes are the conjugacy classes of $C_{4} \times C_{2}^{3}$ and $w=32$. Hence $F\left[C_{4} \times\right.$ $\left.C_{2}^{3}\right] \cong F^{32}$. 
2. If $q \equiv-1 \bmod 4$, then $T=\{1,3\} \bmod 4$. Thus, $p$-regular $F$-conjugacy classes are $\{1\},\left\{a, a^{3}\right\},\left\{a^{2}\right\},\{b\},\{c\},\{d\},\left\{a b, a^{3} b\right\},\left\{a^{2} b\right\},\left\{a c, a^{3} c\right\}$, $\left\{a^{2} c\right\},\left\{a d, a^{3} d\right\},\left\{a^{2} d\right\},\{b c\},\{c d\},\{b d\},\left\{a b c, a^{3} b c\right\},\left\{a^{2} b c\right\},\left\{a c d, a^{3} c d\right\}$, $\left\{a^{2} c d\right\},\left\{a b d, a^{3} b d\right\},\left\{a^{2} b d\right\},\{b c d\},\left\{a b c d, a^{3} b c d\right\},\left\{a^{2} b c d\right\}$ and $w=24$. Hence $F\left[C_{4} \times C_{2}^{3}\right] \cong F^{16} \oplus F_{2}^{8}$.

Hence we have the result.

Theorem 7. Let $F$ be a finite field of characteristic $p>0$ having $q=p^{n}$ elements and $G \cong C_{2}^{5}$.

1. If $p=2$. Then, $U\left(F\left[C_{2}^{5}\right]\right) \cong C_{2}^{31 n} \times C_{2^{n}-1}$.

2. If $p \neq 2$. Then,

$U\left(F\left[C_{2}^{5}\right]\right) \cong C_{p^{n}-1}^{32}$, if $q \equiv 1 \bmod 2$.

Proof. The presentation of $G \cong C_{2}^{5}$ is given by $C_{2}^{5}=<a, b, c, d, e \mid a^{2}=$ $b^{2}=c^{2}=d^{2}=e^{2}=1, a b=b a, b c=c b, d c=c d, e d=d e, e a=a e>$.

1. If $p=2$, then $F G$ will be non-semisimple in this case and $|F|=$ $q=2^{n}$. Since $G \cong C_{2}^{5}$, therefore by Lemma 2 , we have $U(F G) \cong$ $C_{2}^{31 n} \times C_{2^{n}-1}$.

2. If $p \neq 2$, then $|F|=p^{n}$. Using the similar arguments as in Theorem $1, F\left[C_{2}^{5}\right]$ is semisimple and $m=2, \sum_{i=1}^{r}\left[D_{i}: F\right]=32$. By observation we have $q \equiv 1 \bmod 2$ and $t=1$.

Hence $q \equiv 1 \bmod 2$, implies $T=\{1\} \bmod 2$. Thus, $p$-regular $F$-conjugacy classes are the conjugacy classes of $C_{2}^{5}$ and $w=32$. Therefore, $F\left[C_{2}^{5}\right] \cong F^{32}$ and we have the result. 


\section{References}

[1] S. F. Ansari and M. Sahai, "Unit groups of group algebras of groups of order 20", Quaestiones Mathematicae, vol. 44, no. 4, pp. 503-511, 2021.

[2] S. Bhatt and H. Chandra, "Structure of unit group of $\mathrm{F}_{\mathrm{p}^{\mathrm{n}}} \mathrm{D}_{60}$ ", Asian-European Journal of Mathematics, vol. 14, no. 5, Art, ID. 2150075, 2021.

[3] L. Creedon, "The unit group of small group algebras and the minimum counter example to the isomorphism problem", International Journal of Pure and Applied Mathematics, vol. 49, no. 4, pp. 531-537, 2008.

[4] R. A. Ferraz, "Simple components of the center of $F \mathrm{G} / \mathrm{J}(\mathrm{F} G)$ ", Communications in Algebra, vol. 36, no. 9, pp. 3191-3199, 2008.

[5] G. Karpilovsky, Unit groups of classical rings. Oxford: Clarendon Press, 1988.

[6] G. Karpilovsky, Group Representations: Introduction to group representations and characters, vol. 1-B. Amsterdam: North-Holland, 1992.

[7] M. Khan, "Structure of the unit group of FD10", Serdica Mathematical Journal, vol. 35, no. 1, pp. 15-24, 2009.

[8] M. Khan, R. K. Sharma, and J.B. Srivastava, "The Unit Group of $\mathrm{FS}_{4}$," Acta Mathematica Hungarica, vol. 118, no. 1-2, pp. 105-113, 2008.

[9] N. Makhijani, R. K. Sharma, and J. B. Srivastava, "The unit group of algebra of circulant matrices", International Journal of Group Theory, vol. 3, no. 4, pp. 13-16, 2014.

[10] N. Makhijani, R. K. Sharma, and J. B. Srivastava, "The unit group of $\mathrm{F}_{\mathrm{q}}\left[\mathrm{D}_{30}\right]$ ", Serdica Mathematical Journal, vol. 41,no. 2-3, pp.185-198, 2015.

[11] C. P. Milies, and S. K. Sehgal, An introduction to group rings. Dordrecht: Kluwer Academic Publishers, 2002.

[12] M. Sahai and S. F. Ansari, "Unit groups of finite group algebras of Abelian groups of order at most 16", Asian-European Journal of Mathematics, vol. 14, no. 03, Art. ID. 2150030, 2021. 
[13] R. K. Sharma, J. B. Srivastava, and M. Khan, "The unit group of F A4", Publicationes Mathematicae Debrecen, vol. 71, no. 1-2, pp. 21-26, 2007.

[14] G. Tang and Y. Gao,"The unit group of F G of group with order 12", International journal of pure and applied mathematics, vol. 73, pp. 143-158, 2011.

[15] G. Tang, Y. Wei and Y. Li, "Unit groups of group algebras of some small groups", Czechoslovak Mathematical Journal, vol. 64, pp. 149-157, 2014.

\section{Suchi Bhatt}

Department of Mathematics and Scientific Computing,

M. M. M. University of Technology,

Gorakhpur

India

e-mail: 1995suchibhatt@gmail.com

and

\section{Harish Chandra}

Department of Mathematics and Scientific Computing, M. M. M. University of Technology,

Gorakhpur

India

e-mail: hcmsc@mmmut.ac.in

Corresponding author 